\title{
Engineered multifunctional nanowires as novel biosensing tools for highly sensitive detection
}

\author{
Pooja Arora • Annu Sindhu • Neeraj Dilbaghi • \\ Ashok Chaudhury
}

Received: 23 April 2012 / Accepted: 15 June 2012/Published online: 4 July 2012

(C) The Author(s) 2012. This article is published with open access at Springerlink.com

\begin{abstract}
Advances in semiconductive nanowires with novel electronic and optical properties contribute towards new detection methods and revolutionize the way of biosensing. Owing to the large amount of literature pertaining to nanowires (indium oxide, polymer, silicon, and gold), the consequent proposal highlights the research published to feature ultra-sensitive handy biosensors. The focus of this article is the effective evaluation of the outstanding scientific challenges in one-dimensional nanostructures (nanowires) towards innovative and exceptional prospects in designing and fabrication of excellent primary transducers/sensors.
\end{abstract}

Keywords Biosensors - Electronics - Nanostructures · Nanowires $\cdot$ Semicoductor

\section{Introduction}

It is worthwhile mentioning that despite the speedy evolution in the area of nanotechnology, perspective for nanostructure construction towards the implementation of nanoscale interface engineering becomes necessary for addressing challenges like long-term biocompatibility and sensitivity. Nanotechnology is the science of creating functional materials and devices at nanoscale (Poole and Owens 2003) level. It includes methods and techniques for modifying the materials and it also uses nanoscale structures (1-100 nm; Fig. 1) for obtaining novel properties

P. Arora $(\bowtie) \cdot$ A. Sindhu $\cdot$ N. Dilbaghi · A. Chaudhury Department of Bio and Nano Technology, Guru Jambheshwar University of Science and Technology, Hisar 125001,

Haryana, India

e-mail: poojaapr1@gmail.com among the modified materials. One such potential example is the one-dimensional (1D) rigid nanostructure also referred as nanowires. The nanowires have unique metallic (Yin et al. 2001), semiconducting (Wu et al. 2002), and insulating (Sung and Bae 2003) properties. Nanowire fabrication chiefly accommodates metals, metal alloys, carbon, and polymers. Fabrication of a nanowire, which is used as a sensing device structure, involves a set of techniques based on nanotechnology. Nanowire sensors work by converting chemical and biological binding events into electronic and digital signals. Biosensor devices based on nanowires are having great potential in the direct detection of biological as well as chemical species. The extremely high surface-to-volume ratio of 1D biosensor like nanowires and nanotubes makes them ideal building blocks for biosensor development. They have strong biocompatibility and size similarity with the host (biomolecules), with emphasis on novel electron transport properties (Tey et al. 2010). Nanowires can detect even slight disturbances from the surrounding environment (due to their high surface area to volume ratio). The constituent atoms reside on the surface of the nanostructures, which can generate electrical signals even with slight disturbances (Lacerda et al. 2006, 2007; Polizu et al. 2006; Popov et al. 2007) in the system. Therefore, due to electronic conductance, which is being generated by minor changes on surface of nanowires, these can work as real time and label-free bioelectronic devices.

The physical, electronic and chemical properties of biosensors strongly depend on the number and kind of atoms that make up the particle. Nanowires can be readily prepared by electro-deposition into the pores of a membrane through template-directed electrochemical synthesis. Additionally, many methods are employed to synthesize nanowires such as template-aided synthesis, and vaporliquid-solid (VLS) method. From diagnosing disease to the 


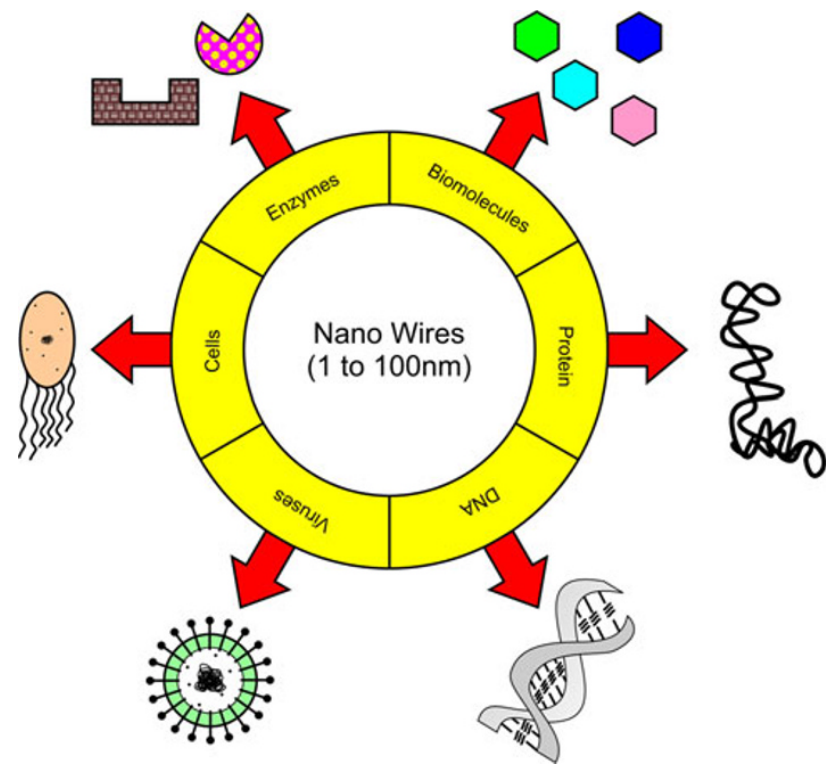

Fig. 1 Tool range of nanowire sensor for detection of biomolecules

drug discovery and screening of new drug molecules, the detection and quantification of biological and chemical species are critical to almost all areas of health care and life sciences. Detection is based on signal transduction, which is associated with the selective recognition of a biological or chemical species of interest. Several approaches like enzyme-linked immune-sorbent assays (ELISA) (Ward et al. 2001), surface plasmon resonance (SPR) (Campagnolo et al. 2004), nanoparticles (Mirkin et al. 1996), chemically sensitive field-effect transistors (CHEMFETs) (Domansky et al. 1993; Janata 1994), microcantilevers (Wu et al. 2001), and carbon nanotubes (Chen et al. 2003, 2004a, b) have been used for the detection of biological molecules but none among these methods of detection has yet demonstrated the combined effect of biomolecules detection. The new era of research nanostructure, such as nanowires (NWs) (Morales and Lieber 1998; Hu et al. 1999; Lieber 2003; Wang 2004) volunteers innovative and exceptional prospects to develop novel biosensors for covering broad perspectives. The diameters of these biosensors are in range of the biological and chemical species. Therefore, these serve as excellent primary transducers (Fig. 2) for producing signals. The biosensor which is chiefly composed of inorganic NWs possess highly reproducible electrical (Lieber 2003; Duan et al. 2001; Cui and Lieber 2001; Gudiksen et al. 2002; Cui et al. 2003; Thelander et al. 2003; Jin et al. 2004; Wu et al. 2004; Zheng et al. 2004) as well as optical (Chan et al. 2002; Alivisatos 2004) properties. A nanowire is a potent agent for the progress of ultra-sensitive, miniaturized sensing micronanodevices.

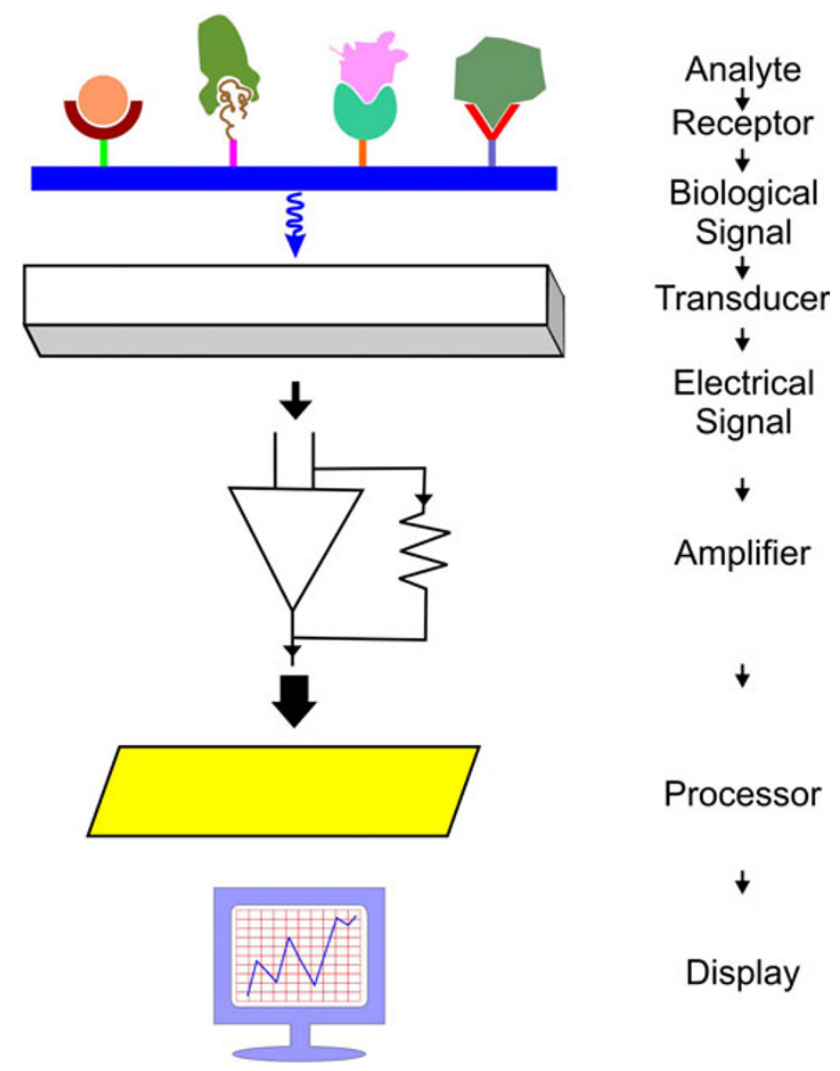

Fig. 2 Schematic representation of working mechanism for the nanowire sensors. Receptors attached aids to nanowire detection of specific biomolecules. When binding occurs biological signal is produced (showed by wavy lines), transducer converts and process it into electrical amplified signal

Indium oxide $\left(\operatorname{In}_{2} \mathrm{O}_{3}\right)$ nanowires

A wide band-gap semiconductor, indium oxide has been extensively used in microelectronics as window heaters, solar cells, gas sensors and liquid-crystal displays ( $\mathrm{Li}$ et al. 1989; Shigesato et al. 1992; Sreenivas et al. 1985). A range of protocols has synthesized indium oxide nanorods and nanowires, such as carbon assisted synthesis, Au-catalyzed VLS growth, direct thermal heating, and solvothermal method (Chen et al. 2004a, b; Kalyanikutty et al. 2005; Nguyen et al. 2007; Peng et al. 2002; Wan et al. 2006; Yu et al. 2003). A well-designed use of indium oxide $\left(\operatorname{In}_{2} \mathrm{O}_{3}\right)$ nanowires has explored in biosensing applications ( $\mathrm{Li}$ et al. 2003). The nanowire surface was treated with tert-butylamine, aminopropyltriethoxy silane (APTES), or butyl nitrite to design $\operatorname{In}_{2} \mathrm{O}_{3}$ nanowire-based field-effect transistor (FET). The amine group donates electron and the nitro group withdraws electron leading to potential change in the conductance of the FET. The adapted $\operatorname{In}_{2} \mathrm{O}_{3}$ nanowires (FET) were exploited to sense various biomolecules such as the cholesterol carrier in blood (LDL cholesterol). 


\section{Polymer nanowires}

Conducting polymers have the mode to manipulate their electrical, magnetic, and optical properties and are very easy to fabricate. It makes them a prospective candidate for sensors. It majorly includes polypyrrole, polyanilinebased nanowires (Heeger 2002; Hurst et al. 2006). A polypyrrole nanowire-based $\mathrm{pH}$ sensor was explored by the studies of Yun et al. (2004). Inside a microfabricated nanochannel, nanowires were engineered through monomer electrochemical polymerization, which behaves like a new electrochemical cell. Manifold nanochannels and electrochemically polymerized pyrrole/aniline production further prepares the array of "numerous independently addressable" nanowires of polypyrrole (PPy) and polyaniline (PANI) (Ramanathan et al. 2004; Wanekaya et al. 2006). In the presence of avidin conjugated with $\mathrm{ZnSe} /$ CdSe quantum dots, it was found that the polymerization process of pyrrole entraps quantum dot conjugates within the nanowires (Ramanathan et al. 2004). A template-free technique was pertained for large array-oriented electrochemical deposition of polyaniline nanowires (Liu and $\mathrm{Ju}$ 2003). Polyaniline nanowires ( $\sim 50$ to $70 \mathrm{~nm}$ diameter) were used to spot hydrogen peroxide $\left(\mathrm{H}_{2} \mathrm{O}_{2}\right)$, though it also pays meticulous interest in enzyme-based biosensing. In contrast, conductive polyaniline nanowires superimpose coating of iron hexacyanoferrate $(\sim 20 \mathrm{~nm}$ diameter $)$ nanoparticles. This aids in sensing of $\mathrm{H}_{2} \mathrm{O}_{2}$ by declining the $\mathrm{H}_{2} \mathrm{O}_{2}$ electro-oxidation potential electrochemically. Thereby, it helps in reducing intrusion from other biological species.

Nucleic acids (DNA) as a template can be used for the making of biological polyaniline nanowires (Ma et al. 2004). The orientations in DNA strands were controlled by thermal oxidization of Si surface, and subsequent incubation in an aniline monomer was thereby controlled through electrostatic interactions. Enzymatic polymerization produces nanofibers with the assistance of associated aniline monomers. The sensors were thus fabricated by an array of gold electrodes and a cross immobilization of DNA strands. In this aspect, polyaniline nanowires were found to be the good substrates for DNA attachment (Chang et al. 2007; Zhu et al. 2006). Conducting polymer nanowirebased sensor of glassy carbon electrode in an electrolyte solution of aniline could directly assemble through electrochemical layer-by-layer deposition protocol. Confining to the advanced electrochemical sensing assets of diamond as transducer material, Nebel et al. (2008) pioneered a novel biosensing platform by geometrically restricted DNA bonding based on perpendicularly aligned diamond nanowires. On the transducer, mesospacing for DNA as biomolecules was created electrochemically, by ultra-hard vertically aligned diamond nano-wires, which were formed from reactive ion etching to construct a well-build biosensor platform for future prospects.

\section{Gold nanowires}

Glucose and hydrogen peroxide nano sensors were composed from gold nanowires by electro-deposition route $(\mathrm{Lu}$ et al. 2007). Modified layer-coated electrode of gold nanowires was fabricated by dispersion within chitosan solution followed by enzyme immobilization to form an infrangible film. Modified electrode thus formed was found 30 times more sensitive than conventional gold electrode. The excellent biocompatibility and large specific surface area of gold nanowires made it an ideal candidate to serve as a new-fangled biosensing platform that provides eventual transaction for the adsorption of enzymes. In a study, the utility of 3D architectural nanowires biosensor was used for very low detection thresholds for target DNA detection (Lapierre-Devlin et al. 2005). The key factor contributing to the elevated sensitivity in DNA-modified nanowires are high signal-to-noise ratio with large electrocatalytic signals than those recorded at bulk gold surfaces. To validate current facts of nano technology experiments based on the enzyme-linked immunosorbent assay various systems are under construction for the development of bacterial detection from biosensors. A Sensor made of gold-coated nanowired structures with glycoconjugate (antibody) is used for the detection of very low bacterial concentration in a couple of minutes (Basu et al. 2004). The octadecane thiol sensor was fabricated with single nanoporous gold nanowires. Liu and Searson (2006) reported adsorption of an alkanethiol with real time to confirm the authenticity of protocol. Nanorods, the lowaspect-ratio nanowires, prepare the surface plasmon technique-dependent dormant biosensors (Niidome et al. 2004). For example, gold nanorods by electrostatic attachment and dispersion in solvents smartly facilitate solvent sensing by chemical synthesis (Niidome et al. 2003). In a recent study, for multiplex detection at biological end, various physical properties of gold nanorods were utilized to develop a sensitive lab-in-a-tube biosensor (Irudayaraj 2007).

Martin et al. (1999) first reported bimetallic or multisegmented gold and platinum nanowires. In their study, they found sequential electro-deposition of gold and platinum metals in the pores of porous alumina membrane. Towards this end, amendments in the surface of nickel and gold nanowires results in selective adsorption of proteins (Birenbaum et al. 2003; Gao et al. 2007). These observations were further elaborated by demonstrating bifunctional and differential protein adsorption in bimetallic nickel and gold nanowires, for the same but hybrid and much stable nanowired structure towards biological molecules (Birenbaum et al. 2003). 
Silicon nanowires

The array of silicon nanowire ( $\mathrm{SiNW}$ ) biosensors has been used for the DNA sensor, as it discriminates well against mismatched targeted DNA. It directly monitors the DNA hybridization event in situ and in real time conditions. One of the studies reported (Wang et al. 2006) a metal-oxide semiconductor compatible fabrication of highly ordered n-type silicon nanowire (SiNW) arrays. Per se in the sensing mechanism of SiNW biosensor, change in charge density on the surface of SiNW can be tacit at the time of post-hybridization, the so-called "field effect". The SiNW array biosensor includes its label-free, sensitive, nonradioactive profile for the development of specific gene expression tools and its clinical applications.

In the route of FET-based sensing, the boron doped (p-type) Si nanowires (SiNWs) fabricated with organobiomolecules lead to change in conductance of the target. The change in conductance of the modified silicon nanowire was found directly proportional to the streptavidin concentration in solutions. A SiNWs FET works as a $\mathrm{pH}$ sensor by fabricating and adapting the gate oxide with charged chemical species. Nanoscale sensors can be fabricated easily using semiconducting materials (like Si for nanowires). Due to the fact, they have the aptitude to manage the doping and simplicity for surface modifications. Consistency and reproducibility of the sensor bends mainly on the eminence of the SiNWs, and the control on the nanowire surface variations (Cui et al. 2001; Kane and Stroock 2007).

Li et al. (2004) developed the sequence-specific SiNWbased sensor. Using silicon as a substrate, doping of SiNWs was performed chiefly by ion implantation method to prepare a handy chip test sensor. Additionally, hybridization technology was employed for the DNA detection, based on the targeted DNA (12-mer oligonucleotide) and the matrix of immobilized probe. Interaction between the complementary DNA and SiNWs alter the conductance variably, thereupon it facilitates the sensing of the oligonucleotides. To confirm the specificity of the sensor, when non-complementary DNA (a base mismatch) was inserted into the sample, no comeback was recorded in the conductance. In a FET device, detection of DNA and DNA mismatches (Fig. 3) was also described using CVD-grown p-type SiNWs (Hahm and Lieber 2004), giving it a completely new roadmap towards handy sensors for future.

\section{Nanowire as bioelectrical device}

Electrical detection of biological binding events is an additional alternative method for detection, over currently used colorimetric and fluorescence-based methods. The

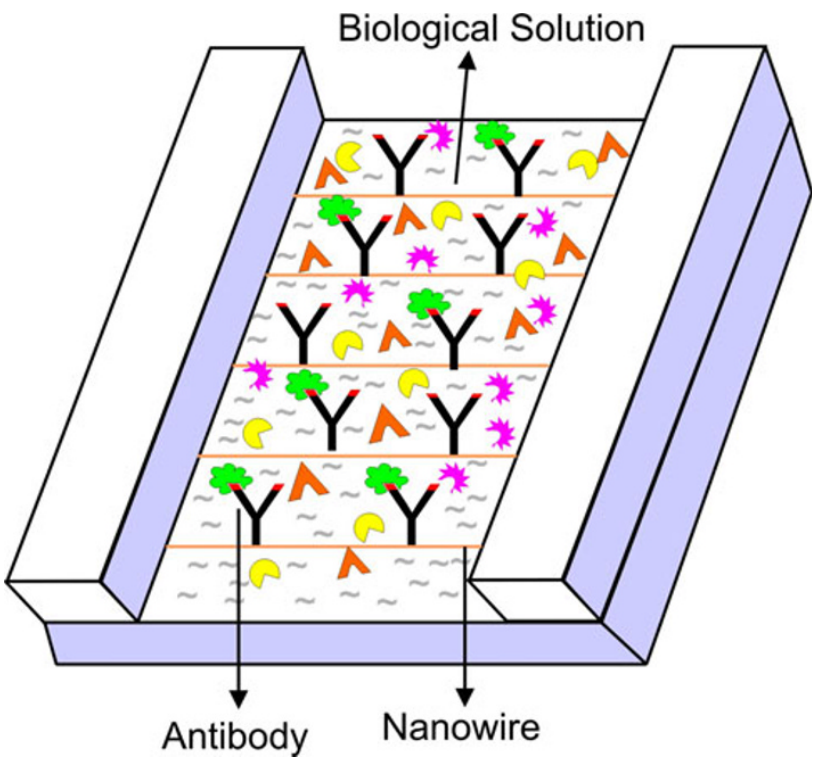

Fig. 3 Detection of proteins (antibody) by nanowires. Antibody $(Y)$ attached to orange nanowire on the nanochip. Its red color indicates active sites at which green colored molecules are binded due to specificity this is physically indicated by there difference in the binding sites

traditional detection methods such as fluorescence-based methods and ELISA are unsuitable for miniaturization and integration into hand-held sensors. Due to lesser response time and high sensitivity, the demand for this technology is rising. Due to its ease to integrate with semiconductor technology, the horizons for the development of nanowirebased detection systems are expanding. In present scenario, detection by analytical biodevices is emerging as tools for monitoring biological and chemical impurities but most of these commercial analytical biodevices are based on optical detection methods, which require heavy power sources, optics and lasers as measuring devices. Optical techniques work on phenomenon of florescence, which requires charge coupled camera (CCD), and photomultiplier tube (PMT). The use of fluorescent technique is not convenient because fluorescent dye may give false results due to photo bleaching effect, with its high expense of experiment. In addition, with optical sensors only micro-nano level of analyte can be monitored (Lee et al. 2010). In contrast to this, the electrical detection-based analytical biodevices are much more sensitive and needed simple instrumentation as well. This lead to fast growing of the electrical detection systems in the field of analytical biodevices and a number of electrical measuring systems with high sensitivity have been developed (Chen et al. 2004a, b; Syvanen and Soderlund 2002; Park et al. 2002, 2005; Willner and Willner 2002; Zheng et al. 2005; Bunimovich et al. 2006; Rawlett et al. 2002; Tsai et al. 2006; Lee et al. 2007). The flow of electrical current is used as the basis for the analysis and 
monitoring of signals from analytes (Fig. 2). As biomolecular recognition in these biosensors are combined with important advantages, such as high sensitivity, low cost, low power requirements and portability, it serves as very attractive method for detection of compounds. Compared to conventional analytical methods, biosensor-based electrical detection systems have higher sensitivity and selectivity. In life sciences and healthcare from diagnosis to treatment, biosensors serve the purpose for rapid detection of biological and chemical species in the host machinery.

The electrical properties of the conducting material (e.g. conductance) can be controlled majorly by the surface contribution. As much more constituting atoms are present on the surface, the electrical conductivity of these nanoparticles raises to higher ratios. Towards this end of nanoparticles, ion selective label-free electronic sensors are attractive in fields for detecting DNA, proteins, and viruses. Silicon has been widely used in the development of biosensors, as it is biocompatible with semiconducting materials (Cui et al. 2001; Patolsky et al. 2004; Wang et al. 2005; Li et al. 2005). The presence of a number of charged biomolecules on the surface of a semiconducting nanowire will induce a drastic change in the nanowire conductance and thus, an active semiconductor nanowire due to the field effect advantage can be used as an ultrasensitive sensor.

\section{Synthesis of nanowires}

Generally, nanowires are usually prepared by either 'topdown' approach or' bottom-up' approach. In "bottom-up" approach the self-assembly of small size structures forms into larger structures while in "top-down" approach, the reduction of large systems into smaller sizes takes place to produce multifunctional nanoscale structures $(\mathrm{Li}$ et al. 2005; Vaseashta and Dimova-Malinovska 2005; Zhang et al. 2005). Top-down approach includes electron-beam lithography or photolithography while bottom-up approach includes synthesis from molecular precursors such as VLS mechanisms. In top-down, the extraction of a nanowire from a bulk sample is done through processes such as electron-beam lithography or mechanical reduction. The mechanical reduction method was found to be more accurate for fabricating nanowires but is time consuming and requires specialized, skilled operators. However, the synthesis of nanowires from bottom-up process is easy and rapid, but it is not that much uniform as the top-down process. For developing nanotubes, nano imprint lithography (NIL) has emerged as a promising technique. Where 'top-down' and 'bottom-up' processes have their own advantages and disadvantages, the NIL process is an advanced hybrid technique which is cost effective, easy to handle, consumes less time and suitable for large area fabrication of nanostructures.

\section{Nanowire field-effect sensors}

In modern electronics, detectors based on the semiconductor nanowires were configured as FET and are among the most widely used biodetection devices. Semiconductor nanowires composed of silicon as well as other material can function as field-effect devices (Lieber 2003; Cui and Lieber 2001; Cui et al. 2003; Thelander et al. 2003; Jin et al. 2004; Wu et al. 2004; Zheng et al. 2004). The conductivity changes in response to variations in the electric field and/or potential at the surface of semiconductor (Lieber 2003; Cui and Lieber 2001; Patolsky and Lieber 2005). The p-type silicon (p-Si) semiconductor is used in standard FET coupled to metal source from where current is injected and subsequently, it drains electrodes through which a current is collected. Current in FET moves in a path from the source to the drain electrode, which is potentially called a "channel". A third electrode known as the gate electrode (McAlpine et al. 2007; Stern et al. 2007) regulates the conductance between the source and the outer drainage of a semiconductor. The separation in path of the gate and charge region via a channel region was coherently induced by inserting insulating oxide layers in an FET structure. When the voltage is applied to the gate, the variation in surface potential begins current flow (due to the change in charge) in a specific direction. The applied gate voltage induces an opposite charge in a conducting region across the insulating oxide layer, which is equivalent to extra charge on an oxide capacitor. In the case of a $\mathrm{p}$-Si or other p-type semiconductor, applying a positive gate voltage reduces the conductance, whereas applying a negative gate voltage leads to increase in the conductance. This happens due to depletion of carriers in former and accumulation of carriers in latter case, respectively. The conductance in this way depends upon the gate voltage, which makes FETs as natural candidates for electricalbased analytical biodevices. This idea for sensing with FETs was introduced several decades ago for CHEMFETs (Domansky et al. 1993; Janata 1994; Bergveld 1972; Blackburn et al. 2001), but the limited sensitivity of previous planar devices has prevented these from having a large impact as chemical or biological sensors.

Sensing mechanism

During fabrication, some specific recognition groups were linked to the surface of semiconductor wires, which aids in analytical measurements at every step. A natural thin silicon oxide layer coated on the surface of nanowires provides the linking-site to the recognition group of molecules present in the analyte. During detection, these linked groups were exposed to the solution containing the biological or chemical molecules. Specific binding occurs

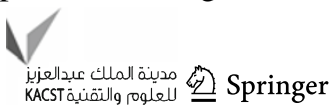


between molecules in solution and molecules linked to the nanowire surface. This leads to some changes in chemical environment of the surface of nanowire and sensed in terms of changes in conductance. This can be demonstrated by taking example of proteins, as proteins possess net negative charge in aqueous solution. After specific binding to the linked molecules on the surface of nanowires, it causes increase in surface negative charge. Increase in surface negative charge will result in ultimate increases in conductance of P-type nanowire devices. These field-effect sensors can detect a broad range of biological as well as chemical species based on surface modification in nanowires with specific receptors (Fig. 2). The varied advantages, namely label-free, biocompatibility with biological molecules, real time direct signaling, high sensitivity, high selectivity, and the potential for arranging multiple detection systems into a highly ordered manner keep detection system of NW biosensors on top to the conventional detection technologies.

\section{Nanowire for detection of proteins}

The first example of electrical detection of nanostructureintegrated protein in solution was reported in biotin that binds specifically with streptavidin (Cui et al. 2001). The biotin, thus linked to the oxide surface of the nanowires, was used as the binding receptor for the analyte, streptavidin. The conductance increased rapidly to a constant value with flooded solutions of the streptavidin. Nanowire sensor devices hence become stable after the addition of pure buffer solution. Nowadays, nanowire devices can be used for the simultaneous detection of multiple proteins (Zheng et al. 2005). This serves as versatile detection platform and had great potential in disease diagnostics (Etzioni et al. 2003) like diagnosis of complex cancers. Effective cancer diagnosis will require rapid analysis of clinically relevant samples, such as blood serum detector, which helps in prognosis from a single drop of blood. The analysis of patterns of multiple cancer markers helps in robust diagnosis of any person within a population (Sidransky 2002). Detection of markers associated with different stages of disease pathogenesis could further facilitate early detection of disease by specific protein biomarkers. By selectively linking different receptors on the different areas of a sensor or an array chip using microarray technique, multiplexed detection of distinct marker proteins can be carried out in highly sensitive and selective manner. For the demonstration of this critical capability of SiNW devices, various studies on monoclonal antibody receptors linked with PSA (NW1), carcinoembryonic antigen (NW2), and mucin-1 (NW3) were reported. Different protein solutions of undiluted human serum were sequentially delivered to the device array and conductance measurements were recorded simultaneously from NW1, NW2 and NW3. Sensitivity of technique goes up to femto molar level and with $\sim 100 \%$ selectivity (Sidransky 2002) to confirm its crucial role in research.

\section{Nanowire as biosensor in solution}

The very first use of nanowire field-effect devices to detect hydrogen ion concentration $(\mathrm{pH})$ of analytes in solution occurred in 2001 (Cui et al. 2001). A p-SiNW device was transformed into a $\mathrm{pH}$ sensor. The original surface of semiconductor surface having silicon oxide surface was modified with 3-aminopropyltriethoxysilane that results in amino groups at the nanowire surface along with the naturally present silanol groups of the oxides. Both these functional groups act as receptors for hydrogen ions. The charge density of the surface nanowire varies depending upon the protonated and deprotonated state of these functional moieties. As the $\mathrm{pH}$ of solution increases, there is comparable and simultaneous stepwise observable increase in the conductance of the biosensor. For a biosensor to be accurate, a linear increase in conductivity with increasing $\mathrm{pH}$ is must. The use of these two different functional groups solves this purpose by acting at different $\mathrm{pH}$ ranges. With increasing conductance, there is decrease in surface positive charge and accumulation of carriers (increase of the negative charge), which "turned on" the p-type FET biosensor device. The key role played by surface receptor in defining the hydrogen-ion response was further tested in nanowire, where the silicon oxide surface layer was not modified with 3-aminopropyltriethoxysilane, by recording the conductance versus $\mathrm{pH}$ for a particular sample. Only the silanol group present on nanowire functions as a receptor for changes in hydrogen ion concentration. In this case, measurements of the conductance as a function of $\mathrm{pH}$ results in the formation of two different response regimes. If $\mathrm{NW}$ surfaces contain both amino and silanol receptors, then at low $\mathrm{pH}(2-6)$, change in conductance gradually diminishes but in contrast becomes larger and comparable at very high $\mathrm{pH}(6-9)$ step. If we compare the $\mathrm{pH}$-dependent changes in conductance to changes in $\mathrm{pH}$ dependent surface charge density derived from silica, the results were found in excellent harmony with each other (Bolt 1957), which reflects that the sensing mechanism was in fact because of field effect and thus, it opens the gateway for the other acidic and basic sensors of versatile importance.

\section{Nanowire as tool for drug discovery}

In heart of the identification of organic molecules, development of new pharmaceuticals and elucidating complex 
pathways in biological systems lays a phenomenon of the specific binding of proteins to the biosensors (Strausberg and Schreiber 2003). The identification of molecular inhibitors of tyrosine kinase was carried out using this approach. The proteins that mediate signal transduction in mammalian cells by phosphorylation of a tyrosine residue of the substrate protein using adenosine triphosphate as a phosphate group donor are known as tyrosine kinase (ATP) (Becker 2004). Deregulation of the phosphorylation process may lead to several diseases, including cancer (Becker 2004). For the screening of phosphorylation inhibitors, NW surface was effectively employed to link the Ab1 tyrosine kinase. It leads to simultaneous monitoring for the binding of ATP molecules and the competitive inhibition of ATP binding with organic molecules (same as the role of drug Gleevec) (Wang et al. 2005). The binding of the negatively charged ATP to Ab1 linked at the SiNW surface was detected as an increase in the conductance of p-type NW device and data recorded from Ab1-modified p-SiNW devices showed reversible, concentration-dependent increases in conductance upon introduction of ATP solutions containing different small molecules as inhibitors. The increases in conductance were consistent with the binding of negatively charged ATP to Ab1 (Wang et al. 2005). Greater importance was given for the ability to quantify inhibition of ATP binding by drug molecules. The normalized conductance plots recorded from Ab1-modified p-SiNW devices showed reversible decrease in conductance because of competitive inhibition of ATP binding molecules. This shows substantial advantages of NW detectors over existing conventional methods. It concludes that only NW detectors include direct and high-sensitivity analysis of binding and inhibition with a minimum amount of protein receptor in very less time duration.

\section{Nanowire for virus detection}

To define the ultimate sensitivity of nanowire FET devices the detection of viruses is carried out using these biosensors which are among the most important causes of human disease and are agents for biological warfare and terrorism (Stadler et al. 2003; Atlas 2003). The ultimate limit of detection, achieved by the use of these biosensors is to detect one single entity reliably. Specific binding of virus particle to the antibody receptor results in changes of the conductance from the baseline source. However, when it unbinds from antibody receptor, the conductance will return to the baseline. When highly dilute influenza A virus solution (Bolt 1957) (viruses $/ \mathrm{mL}$ ) was delivered to $\mathrm{p}$-SiNW devices which are modified with monoclonal antibody (specific for influenza A virus), distinct conductance changes were observed. This is the characteristic output of the binding and unbinding of a single positively charged influenza particle (Patolsky et al. 2004). It was further been confirmed by simultaneous electrical and optical measurements with fluorescently labeled influenza virus. For an electrical response to be obtained in harmony, the virus must have to be in contact with the nanowire device. Now research for devising of ultra-dense nanowire arrays is in progress, where the minimum scale could be set by the size of the virus.

\section{Nanowire as DNA detective}

Biological molecules (nucleic acids and proteins) carry charge in aqueous solutions. The surface activation of NWs with specific receptors stimulates detection of charged biomolecules. DNA-modified gold nanowires and silicon nanowires have been used for the DNA sensor. For the detection of single-stranded DNA present in biological samples, SiNW field-effect devices are used. Peptide nucleic acids (PNAs) (Jensen et al. 1997) were used as the receptor for detecting the presence of DNA in biological samples. As the uncharged PNA, molecules have greater affinity and stability towards single-stranded DNA (Fig. 4). The binding of negatively charged polyanionic macromolecule to PNA causes increase in conductance of p-type nanowire (Hahm and Lieber 2004). PNA receptor was designed for cystic fibrosis transmembrane receptor gene. These recognize the wild-type sequence versus the $\Delta \mathrm{F} 508$ mutation site in the transmembrane receptor gene. When 60-fM wild-type DNA sample solution was added in p-SiNW device, consistent increase in conductance was observed. This was the result of increase in negative surface charge density, which is associated with the binding of a negatively charged DNA at the surface of modified nanowire. However, the sequences having $\Delta$ F508 mutation did not show this stable change in conductance (Hahm and Lieber 2004). This was the specific demonstration for the use of nanowire in detection of diseases associated with genetic modifications within genome. The potential of nanowire arrays as activity-based diagnostic tool is

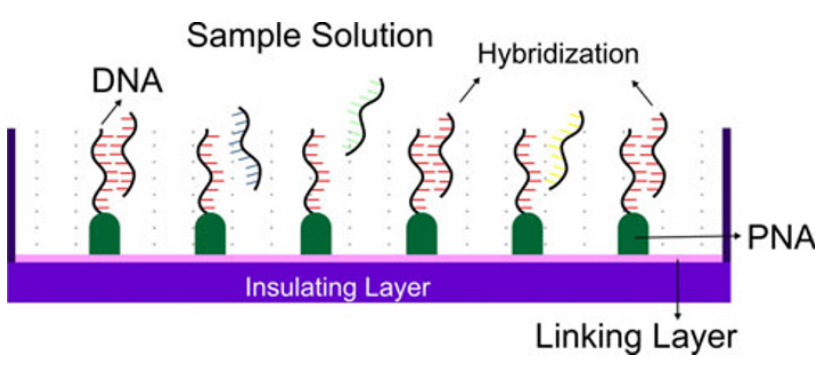

Fig. 4 Nanowire sensor for DNA/PNA detection. Only complementary DNA hybridizes 
illustrated by detection of the activity and inhibition of telomerase, which is inactive in normal somatic cells, but active in $80 \%$ of known human cancers and it adds TTAGGG repeat sequence to chromosomal ends (Zheng et al. 2005; Moyzis et al. 1998; O’Reilly et al. 1999). For cancer detection as well as treatment, telomerase serves as a potential marker. The assay detects the presence or absence of telomerase simply by monitoring the changes in conductance of nanowire after delivery of cell extract to the device array (Hahm and Lieber 2004). Addition of deoxynucleotide triphosphates (dNTPs) causes increase in conductance of nanowires due to the presence of negatively charged nucleotides near the surface of device, which further facilitates monitoring. When HeLa cell extract was delivered to the p-SiNWs modified with oligonucleotide primers, they showed well-defined conductance decrease, corresponding to the selective binding of the positively charged telomerase at the surface of the nanowires. It reflects the specificity of positive-signals to the presence of active telomerase in the cell extract with response to modified nanowires (Hahm and Lieber 2004).

\section{Conclusion}

The unique properties possessed by nanowires such as mechanical stability, low-weight, and ability of current enhancement, reduction in potential, biocompatibility with biological and chemical species designate them among the efficient tools for biodetection. The real value of sensors lies in their detection limit range and ultra sensitivity. Their excellent electrical, thermal, magnetic and optical properties provide surplus benefits in their adaptability. The overall properties of biosensors will make these nanowirebased sensors more prominent among researchers and in market within few more coming years. Most of the applications described in the article are concerned with pushing the future-limits of nanowire-based biosensors to detect few molecules or even up to single molecule in a given volume of biological solution.

Open Access This article is distributed under the terms of the Creative Commons Attribution License which permits any use, distribution, and reproduction in any medium, provided the original author(s) and the source are credited.

\section{References}

Alivisatos P (2004) The use of nanocrystals in biological detection. Nat Biotechnol 22:47-52

Atlas RM (2003) Bioterrorism and biodefence research: changing the focus of microbiology. Nat Rev Microbiol 1:70-74

Basu M, Seggerson S, Henshaw J, Jiang J, Cordona RDA, Lefave C, Boyle PJ, Miller A, Pugia M, Basu S (2004) Nano-biosensor development for bacterial detection during human kidney infection: use of glycoconjugate-specific antibody-bound gold nanowire arrays (GNWA). Glycoconjug J 21:487-496

Becker J (2004) Signal transduction inhibitors-a work in progress. Nat Biotechnol 22:15-18

Bergveld P (1972) IEEE development of an ion-sensitive solid-state device for neurophysical measurements. Trans Biomed Eng 19:342-347

Birenbaum NS, Lai BT, Chen CS, Reich DH, Meyer GJ (2003) Selective noncovalent adsorption of protein to bifunctional metallic nanowire surfaces. Langmuir 19:9580-9585

Blackburn GF, Turner AP, Ward AM, Catto JWF, Hamdy FC (2001) Prostate specific antigen: biology, biochemistry and available commercial assays. Ann Clin Biochem 38:633-651

Bolt GH (1957) Determination of the charge density of silica sols. J Phys Chem 61:1166-1170

Bunimovich AL, Shin YS, Yeo W-S, Amori M, Kwong G, Heath JR (2006) Quantitative real-time measurements of DNA hybridization with alkylated nonoxidized silicon nanowires in electrolyte solution. J Am Chem Soc 128:16323-16331

Campagnolo C, Meyers KJ, Ryan T, Atkinson RC, Chen YT, Scanlan MJ, Ritter G, Old LJ, Batt CA (2004) Real-Time, label-free monitoring of tumor antigen and serum antibody interactions. J Biochem Biophys Methods 61:283-298

Chan WCW, Maxwell DJ, Gao XH, Bailey RE, Han MY, Nie SM (2002) Luminescent QDs for multiplexed biological detection and imaging. Curr Opin Biotechnol 13:40-46

Chang Z, Zhu NN, Zhao K, Fan H, He PG, Fang YZ (2007) Study of polyaniline nanowire modified electrode for electrochemical DNA biosensor. Acta Chim Sinica 65:135-139

Chen RJ, Bangsaruntip S, Drouvalakis KA, Wong Shi Kam N, Shim M, Li Y, Kim W, Utz PJ, Dai H (2003) Non-covalent functionalization of carbon nanotubes for highly specific electronic biosensors. Proc Natl Acad Sci USA 100:49844989

Chen RJ, Choi HC, Bangsaruntip S, Yenilmez E, Tang XW, Wang Q, Chang YL, Dai HJ (2004a) An investigation of the mechanisms of electronic sensing of protein adsorption on carbon nanotube devices. J Am Chem Soc 126:1563-1568

Chen YQ, Jiang J, Wang B, Hou JG (2004b) Synthesis of tin-doped indium oxide nanowires by self-catalytic VLS growth. Phys D Appl Phys 37:3319-3324

Cui Y, Lieber CM (2001) Functional nanoscale electronic devices assembled using silicon nanowire building blocks. Science 291:851-853

Cui Y, Wei Q, Park H, Lieber CM (2001) Nanowire nanosensors for highly sensitive and selective detection of biological and chemical species. Science 293:1289-1292

Cui Y, Zhong Z, Wang D, Wang WU, Lieber CM (2003) High performance silicon nanowire field effect transistors. Nano Lett 3:149-152

Domansky K, Janata J, Josowicz M, Petelenz D (1993) Present state of fabrication of chemically sensitive field effect transistors. Analyst 118:335-340

Duan XF, Huang Y, Cui Y, Wang J, Lieber CM (2001) Indium phosphide nanowires as building blocks for nanoscale electronic and optoelectronic devices. Nature 409:66-69

Etzioni R, Urban N, Ramsey S, McIntosh M, Schwartz S, Reid B, Radich J, Anderson G, Hartwell L (2003) The case for early detection. Nat Rev Cancer 3:243-252

Gao Z, Agarwal A, Trigg AD, Singh N, Fang C, Tung CH, Fan Y, Buddharaju KD, Kong J (2007) Silicon nanowire arrays for label-free detection of DNA. Anal Chem 79:3291-3297

Gudiksen MS, Lauhon LJ, Wang JF, Smith DC, Lieber CM (2002) Growth of nanowire superlattice structures for nanoscale photonics and electronics. Nature 415:617-620 
Hahm J, Lieber CM (2004) Direct ultrasensitive electrical detection of DNA and DNA sequence variations using nanowire nanosensors. Nano Lett 4:51-54

Heeger AJ (2002) Semiconducting and metallic polymers: the forth generation of polymeric metals. Synth Metals 125:23-42

Hu JT, Odom TW, Lieber CM (1999) Chemistry and physics in one dimension: synthesis and properties of nanowires and nanotubes. Acc Chem Res 32:435-445

Hurst SJ, Payne EK, Qin L, Mirkin CA (2006) Multisegmented onedimensional nanorods prepared by hard-template synthetic methods. Angew Chem 118:2738-2759

Irudayaraj $\mathrm{CYu}$ (2007) Multiplex biosensor using gold nanorods. J Anal Chem 79:572-579

Janata J (1994) Twenty years of ion-selective field-effect transistors. Analyst 119:2275-2278

Jensen KK, Ørum H, Nielsen PE, Nordén B (1997) Hybridization kinetics of peptide nucleic acids (PNA) with DNA and RNA studied with BIAcore technique. Biochemistry 36:5072-5077

Jin S, Whang D, McAlpine MC, Friedman RS, Wu Y, Lieber CM (2004) Scalable interconnection and integration of nanowire devices without registration. Nano Lett 4:915-919

Kalyanikutty PK, Gundiah G, Edem C, Govindaraj A, Rao RNC (2005) Undoped and doped ITO nanowires. Chem Phys Lett 408:389-394

Kane RS, Stroock AD (2007) Nanobiotechnology: protein-nanomaterial interactions. Biotech Prog 23:316-319

Lacerda L, Bianco A, Prato M, Kostarelos K (2006) Carbon nanotubes as nanomedicines: from toxicology to pharmacology. Adv Drug Deliv Rev 58:1460-1470

Lacerda L, Raffa S, Prato M, Bianco A, Kostarelos K (2007) Cell penetrating CNTs for delivery of therapeutics. Nano Today 2:38-43

Lapierre-Devlin MA, Asher CL, Taft BJ, Gasparac R, Roberts MA, Kelley SO (2005) Amplified electrocatalysis at DNA-modified nanowires. Nano Lett 5:1051-1055

Lee BY, Kim YW, Lee DJ, Hong S (2007) Biotin streptavidin binding detection by single-wall carbon nanotube network junctions fabricated with surface programmed assembly method. BioChip J $1: 76-80$

Lee JH, Oh B-K, Choi B, Jeong S, Choi J-W (2010) Electrical detection-based analytic biodevice technology. BioChip J $4(1): 1-8$

Li X, Wanlass MW, Gessert TA, Emery KA, Coutts TJ (1989) High efficiency indium tin oxide/indium phosphide solar cells. Appl Phys Lett 54:2674-2676

Li J, Xiao LT, Zeng GM, Huang GH, Shen GL, Yu RQ (2003) Amperometric immunosensor based on polypyrrole/poly (m-pheylenediamine) multilayer on glassy carbon electrode for cytokinin N6-(Delta2-isopentenyl) adenosine assay. Anal Biochem 321:89-95

Li J, Lu C, Maynor B, Huang S, Liu J (2004) Controlled growth of long GaN nanowires from catalyst patterns fabricated by "dippen" nanolithographic techniques. Chem Mater 16:1633-1636

Li Z, Rajendran B, Kamins TI, Li X, Cheng Y, Stanley Williams R (2005) Silicon nanowires for sequence-specific DNA sensing: device fabrication and simulation. Appl Phys A Mater Sci Process 80:1257-1263

Lieber CM (2003) Nanoscale science and technology: building a big future from small things. MRS Bull 28(7):486-491

Liu S, Ju H (2003) Reagentless glucose biosensor based on direct electron transfer of glucose oxidase immobilized on colloidal gold modified carbon paste electrode. Biosens Bioelectron 19:177-180

Liu Z, Searson PC (2006) Single nanoporous gold nanowire sensors. J Phys Chem B 110:4318-4322
Lu Y, Yang M, Qu F (2007) Enzyme-functionalized gold nanowires for the fabrication of biosensors. Bioelectrochemistry 71:211-216

Ma YF, Zhang JM, Zhang GJ, He HX (2004) Polyaniline nanowires on Si surfaces fabricated with DNA templates. J Am Chem Soc 126:7097-7101

Martin BR, Dermody DJ, Reiss BD, Fang M, Lyon LA, Natan MJ (1999) Orthogonal self-assembly on colloidal gold-platinum nanorods. Adv Mater 11:1021-1025

McAlpine MC, Ahmad H, Wang D, Heath JR (2007) Highly ordered nanowire arrays on plastic substrates for ultrasensitive flexible chemical sensors. Nat Mater 6:379-384

Mirkin CA, Letsinger RL, Mucic RC, Storhoff JJ (1996) A DNAbased method for rationally assembling nanoparticles into macroscopic materials. Nature 382:607-609

Morales AM, Lieber CM (1998) A laser ablation method for synthesis of crystalline semiconductor nanowires. Science 279:208-211

Moyzis RK, Buckingham JM, Cram LS, Dani M, Deaven LL, Jones MD, Meyne J, Ratliff RL, Wu JR (1998) A highly conserved repetitive DNA sequence, (TTAGGG)n, present at the telomeres of human chromosomes. Proc Natl Acad Sci USA 85:6622-6626

Nebel CE, Yang N, Uetsuka H, Osawa H, Tokuda N, Williams O (2008) Diamond nano-wires, a new approach towards next generation electrochemical gene sensor platforms. Diam Relat Mater 18:910-917

Nguyen P, Ng HT, Kong J, Cassell MA, Quinn R, Li J, Han J, McNeil M, Meyyappan M (2007) Epitaxial directional growth of indiumdoped tin oxide nanowires arrays. Nano Lett 3:925-929

Niidome Y, Nishioka K, Kawasaki H, Yamada S (2003) Rapid synthesis of gold nanorods by the combination of chemical reduction and photoirradiation processes. Chem Commun 18:2376-2377 (Cambridge, England)

Niidome Y, Takahashi H, Urakawa S, Nishioka K, Yamada S (2004) Immobilization of gold nanorods on the glass substrate by the electrostatic interactions for localized plasmon sensing. Chem Lett 33:454-455

O'Reilly M, Teichmann SA, Rhodes D (1999) Telomerases. Curr Opin Struct Biol 9:56-65

Park SJ, Taton TA, Mirkin CA (2002) Array-based electrical detection of DNA with nanoparticle probes. Science 295:1503-1506

Park JW, Jung HS, Lee HY, Kawai T (2005) Electrical recognition of label-free oligonucleotides upon streptavidin-modified electrode surfaces. Biotechnol Bioprocess Eng 10:505-509

Patolsky F, Lieber CM (2005) Nanowire nanosensors. Mater Today 8:20-28

Patolsky F, Zheng G, Hayden O, Lakadamyali M, Zhuang X, Lieber CM (2004) Electrical detection of single viruses. Proc Natl Acad Sci USA 101:14017-14022

Peng XS, Meng GW, Wang XF, Wang YW, Zhang J, Liu X, Zhang LD (2002) Synthesis of oxygen-deficient indium-tin-oxide (ITO) nanofibres. Chem Mater 14:4490-4493

Polizu S, Savadogo O, Poulin P, Yahia L (2006) Applications of carbon nanotubes-based biomaterials in biomedical nanotechnology. J Nanosci Nanotechnol 6:1883-1904

Poole CP, Owens FJ (2003) Introduction to nanotechnology. Wiley, NJ, pp 10-11

Popov AM, Lozovik YE, Fiorito S, Yahia L (2007) Biocompatibility and applications of carbon nanotubes in medical nano robots. Int J Nanomed 2:361-372

Ramanathan K, Bangar MA, Yun M, Chen W, Mulchandani A, Myung NV (2004) Individually addressable conducting polymer nanowires array. Nano Lett 4:1237-1239

Rawlett AM, Hopson TJ, Nagahara LA, Tsui RK, Ramachandran GK, Lindsay SM (2002) Electrical measurements of a dithiolated

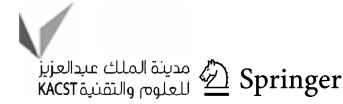


electronic molecule via conducting atomic force microscopy. Appl Phys Lett 81:3043-3045

Shigesato Y, Takaki S, Haranoh T (1992) Electrical and structural properties of low resistivity tin-doped indium oxide films. J Appl Phys 71:3356-3364

Sidransky D (2002) Emerging molecular markers of cancer. Nat Rev Cancer 2:210-219

Sreenivas K, Sudersena Rao T, Mansingh A (1985) Preparation and characterization of rf sputtered indium tin oxide films. J Appl Phys 57:384-392

Stadler K, Masignani V, Eickmann M, Becker S, Abrignani S, Klenk HD, Rappuoli R (2003) SARS—beginning to understand a new virus. Nat Rev Microbiol 1:209-218

Stern E, Klemic JF, Routenberg DA, Wyrembak PN, Turner-Evans DB, Hamilton AD, LaVan DA, Fahmy TM, Reed MA (2007) Label-free immunodetection with CMOS-compatible semiconducting nanowires. Nature 445:519-522

Strausberg RL, Schreiber SL (2003) From knowing to controlling: a path from genomics to drugs using small molecule probes. Science 300:294-295

Sung WJ, Bae YH (2003) A glucose oxidase electrode based on polypyrrole with polyanion/PEG/enzyme conjugate dopant. Biosens Bioelectron 18:1231-1234

Syvanen AC, Soderlund H (2002) DNA sandwiches with silver and gold. Nat Biotechnol 20:349-350

Tey JN, Wijaya IPM, Wei J, Rodriguez I, Mhaisalkar SG (2010) Nanotubes-/nanowires-based, microfluidic-integrated transistors for detecting biomolecules. Microfluid Nanofluid. doi: 10.1007/s10404-010-0640-1

Thelander C, Martensson T, Bjork MT, Ohlsson BJ, Larsson MW, Wallenberg LR, Samuelson L (2003) Single-electron transistors in heterostructure nanowires. Appl Phys Lett 83:2052-2054

Tsai CY, Chang T-L, Kuo L-S, Chen P-H (2006) Detection of electrical characteristics of DNA strands immobilized on selfassembled multilayer gold nanoparticles. Appl Phys Lett 89:203902(1)-203902(3)

Vaseashta A, Dimova-Malinovska D (2005) Nanostructured and nanoscale devices, sensors and detectors. Sci Tech Adv Mater 6:312-318

Wan Q, Wei M, Zhi D, MacManus-Driscoll FL, Blamire MG (2006) Tin-doped indium oxide nanowire arrays communication. Adv Mater 18:234-238

Wanekaya AK, Chen W, Myung NV, Mulchandani A (2006) Nanowire-based electrochemical biosensors. Electroanalysis 18:533-550
Wang ZL (2004) Nanostructures of zinc oxide. Mater Today $7(6): 26-33$

Wang WU, Chen C, Lin K, Fang Y, Lieber CM (2005) Label-free detection of small-molecule-protein interactions by using nanowire nanosensors. Proc Natl Acad Sci USA 102:3208-3212

Wang YD, Zang KY, Chua SJ, Sander MS, Tripathy S, Fonstad CG (2006) Nanodots, and nanoarrows fabricated by a templateassisted approach. J Phys Chem B 110:11081-11187

Ward AM, Catto JWF, Hamdy FC (2001) Prostate specific antigen: biology, biochemistry and available commercial assays. Ann Clin Biochem 38:633-651

Willner I, Willner B (2002) Functional nanoparticle architectures for sensoric, optoelectronic, and bioelectronic applications. Pure Appl Chem 74:1773-1783

Wu GH, Datar RH, Hansen KM, Thundat T, Cote RJ, Majumdar A (2001) Bioassay of prostate-specific antigen (PSA) using microcantilevers. Nat Biotechnol 19:856-860

Wu Y, Yan H, Huang M, Messer B, Song JH, Yang P (2002) Inorganic semiconductor nanowires: rational growth, assembly, and novel properties. Chem Eur J 8:1260-1268

Wu Y, Xiang J, Yang C, Lu W, Lieber CM (2004) Single-crystal metallic nanowires and metal/semiconductor nanowire heterostructures. Nature 430:61-65

Yin AJ, Li J, Jian W, Bennett AJ, Xua JM (2001) Fabrication of highly ordered metallic nanowire arrays by electrodeposition. Appl Phys Lett 79:1039-1041

Yu D, Wang D, Yu W, Qian Y (2003) Synthesis of ITO nanowires and nanorods with corundum structure by a co-precipitationanneal method. Mater Lett 58:84-87

Yun M, Myung NV, Vasquez R, Lee C, Menke E, Penner RM (2004) Extraordinary mobility in semiconducting carbon nanotubes. Nano Lett 4:419-422

Zhang XL, Wang JX, Wang Z, Wang SC (2005) Improvement of amperometric sensor used for determination of nitrate with polypyrrole nanowires modified electrode. Sensors 5:580-593

Zheng GF, Lu W, Jin S, Lieber CM (2004) Synthesis and fabrication of high-performance n-type silicon nanowire transistors. Adv Mater 16:1890-1894

Zheng G, Patolski F, Cui Y, Wang WU, Lieber CM (2005) Multiplexed electrical detection of cancer markers with nanowire sensor arrays. Nat Biotechnol 23:1294-1301

Zhu NN, Chang Z, He PG, Fang YZ (2006) Electrochemically fabricated polyaniline nanowire modified electrode for voltammetric detection of DNA hybridization. Electrochim Acta $51: 3758-3762$ 